\title{
The Implementation of Performance Measurement System (PMS): Malaysian Facilities Management (FM) Industry
}

\author{
N.E. Myeda ${ }^{1}$, S.M. Zaid ${ }^{2}$, R. Sulaiman ${ }^{3}$, N. Mahyuddin ${ }^{4}$ \\ ${ }^{1}$ Bartlett School of Graduate Studies, University College London, WC1H 0NN London, United \\ Kingdom \\ ${ }^{1,2,3,4}$ Faculty of Built Environment, University of Malaya, 50603 Kuala Lumpur, Malaysia
}

\begin{abstract}
Performance Measurement System (PMS) is an effective performance measurement tool and technique that is being widely implemented in the global industries. Literature has suggested the significant contributions of its implementation in enhancing the strategic service delivery and performance. However, there is little study undertaken to explore the PMS implementation in Facilities Management (FM) industry, particularly focusing on Malaysia. This study explores the PMS practice among FM practitioners and their knowledge in Performance Measurement (PM) generally. Findings from this study also proposed the 20 contributing factors that the FM practitioners believed are the barriers in implementing PMS. This research also suggests the future research opportunities in developing a PMS framework that can be used as guidance for FM service delivery in Malaysia.
\end{abstract}

\section{Introduction}

Performance Measurement (PM) is synonym with the process of quantifying action, where measurement is the process of quantification and action correlates with performance [1],[2]. Similarly, it is also defined as the process by which a company manages its performance, in which it should be in line with its corporate and functional strategies and objectives [3]. Kagioglou et al. [4] believes that PM can be used as a tool in determining how successful organisations or individuals have been in attaining their objectives and strategies. This is as it enables managers to make decisions based on facts rather than on assumptions [5]. An operational performance measurement system (PMS) also acts like an early-warning system where it gives an indication for problems and areas for continuous improvement; therefore it has been given a prominent place in most organizations [2],[6],[7].

In literature, PM is a subject that is often discussed but rarely defined, however it has become a very popular research topic since 1980s [8]. Since then, it has received considerable attention and that remarkable progress has been made over recent years. Measurement in particular, is an area that has been discussed increasingly over the past few years with the familiar perceptions like " you can't manage what you can't measure" and "what gets measured gets done" [9]. Measurement is seen essential in providing feedback, builds understanding and encourages intrinsic motivation rather than a tool for top-down management control [10]. Meekings [10] further added that the recognition of measurement in the world industry has shown that the focus was on systematic thinking, fundamental structural change and organisational learning, instead of mindless target-setting, continual fire-fighting or the rigorous allocation of blame. Based on the important element of measurement, PM is often seen 
as an effective tool to increase the competitiveness and profitability of manufacturing companies through the support and encouragement of productivity improvements. According to Cain [11], PM is used as the first stage of any improvement process, which benefits the end users with lower prices and the organisations with higher profit margins and at the same time, enhancing the product quality, and PM with appropriate metrics can quantify both the efficiency and effectiveness of action [1].

PMS has started around the 1860s and 1870s (1). They were first developed based on simple yet straight-forward objectives that were to monitor and maintain the organisational processes aiming to achieve the goals and objectives of the organisations [12]. It is also seen as the comparison of results against expectations with the implied objective of learning to do better [13]. PMS are compared in terms of how easily they are derived from strategic objectives, how easy they are to understand and also whether they help provide a long-term view of performance [8].

However, there are negative effects from PM that are based on complicated and excessive performance measures [6]. This has caused problems with investment and the commitment of people involved through the consumption of a lot of time and the limiting of the freedom of managers due its rigidity and inflexibility. Therefore, a PMS that has sound and appropriate performance measurements is most likely to maximise potential positive benefits. According to Tangen [14], in order for PM to be effective, the measurements must be derived from strategic objectives to ensure that employee behaviour is consistent with corporate goals. They also must provide timely, relevant and accurate feedback, from both a long-term and short-term perspective and should be undertaken in ways that are easily understood by those whose performance is being evaluated.

Previous research normally focuses only on the content of strategic PMS for other industries in general, but not specifically on Facilities Management (FM). The requirements identified for effective measures of PMS are mostly suitable to cater for manufacturing industries. In 1998, Cotts defined Facilities Management (FM) as a managerial practice that integrates the principles of business administration, architecture, and the behavioural and engineering sciences to ensure effectiveness of all these procedures. It exists to support the core business that is the preliminary goal-seeking activities of the enterprise [9]. She further signifies the role of FM in facilitating organisational performance and thereby in providing competitive advantage is widely acknowledged. FM is gaining an increasing importance in the sector of business process outsourcing, but stemmed from the practitioners' experience, there is a lack of a significant theoretical foundation based on empirical evidences about the PM [15].

PM indicates where the organisation is heading; therefore it is also perceived as the language of process for one organisation [16]. Performance relates to building's ability to contribute to fulfilling the functions of its intended use [17]. In order for PM to function effectively, the emphasis must be given to the organisation, process and also job or performer itself [18]. However, Pintelon and Van Puyvelde [19] argue that for service operation, the performance will depend on the perspective applied for instance accountants will think of maintenance in term of costs, top management often is only interested in budget performance, engineers will focus on techniques, production will see performance in terms of equipment availability and support responsiveness.

Despite the different perspectives among the management personnels and officers involved, it is observed that companies using an integrated balanced performance measurement system perform better than those that do not measure their performance [20];[21]. FM can be strategic in managing business support functions and operational, concentrating on the detailed operational activities of the organisation [22]. It also embraces every part of organisation activities, and can be seen as a series of inter-related activities involving the co-ordination of all efforts relating to planning, designing and managing an organisation's physical resources [23]. Facilities represent a substantial percentage of most organisation's assets and their operating costs; thus it is hardly surprising that performance appraisal in FM is becoming a formal and regular part of the FM process [24]. 


\section{Research Approach}

\subsection{Research Aims and Objectives}

There is a lack of systematic process for determining appropriate measurements where an exploratory approach to the area of PM in FM organisations was preferred one as the problem of lack of construct validity is general rather than specific [9]. Hudson et al., [25] also believes that current research in the general area of PM is inadequate in respect of the specific SME context. Most research in FM focuses on the role of the corporate real estate function, outsourcing decisions or more general management issues [26]. Previous studies in performance tend to measure profitability, economics and environmental issues and recently issues on sustainability but there is lacking of studies that focus on performance purely from the FM perspective [27]. There is a causal link between FM practices and performance, prompting the evaluation researchers to question whether performance evaluation does in fact add value and enhance organisational performance [24]. There is also limited KPIs that can be used in FM, the framework tested based on the measures from other indicators are too general for FM service specification [27]. Managers may need to take a wider look at the stakeholders of the organisation, and their various priorities, for a richer understanding of these ambiguous concepts [28]. Although the area of PM is not new, the constructs are neither well-established nor standardised across and even within FM disciplines giving an abundance area for investigation. Therefore, there is a need to determine, verify and integrate the axioms of modern PM in the context of FM Amaratunga [9].

This research aims to explore the PMS in FM particularly in the context of Malaysian industry. Therefore, it seeks to understand the current knowledge and implementation of PMS by the FM to get an overview of the PMS implementation by the FM practitioners.

\subsection{Methodology}

A questionnaire survey was conducted to the FM practitioners in Malaysia, with 37 respondents responded to the survey that comprises of both structured and open-ended questions. The, respondents were asked on the background information, and their knowledge in FM, Strategic FM and also the implementation of PMS in FM. The PMS section seeks to investigate the implementation of PMS by the respondents and its background; the service improvement resulted from the PMS and also to get the respondents' opinions on the barriers or problems in the implementation of PMS. The questionnaires survey adopt both attitude rating scales that is simple attitude scaling (i.e Yes, No and Not Sure) and Likert Scale ranging from very negative to very positive is used to allow respondents to indicate their level of agreements/ perceptions (i.e Highly Disagree to Highly Agree).

\section{Results and Discussions}

\subsection{Respondents Background}

A total of 37 responses were received from the online survey including 2 of which are incomplete. All 35 respondents of the survey are from Malaysia and are all FM practitioners with 3 different job titles; FM executives (60\%), FM manager (28.6\%) and also FM assistant manager $(11.4 \%)$ as stated in. Most of them work in a large (48.6\%) and medium organisations (40\%) while only $11.4 \%$ were in small organisations. The majority of the FM practitioners have been involved in FM industry for more than 3 years $(71.5 \%)$. 


\subsection{Strategic FM}

Respondents were also requested to select the level of FM implementation being practised by their respective companies. $63 \%$ of them are using Operational FM while only $37 \%$ are using Strategic FM .This shows that traditional method is still widely adopted in Malaysia. Further analysis on the 13 respondents that are practicing Strategic FM shows that majority of them $(69 \%)$ agree that strategic FM improves their service quality and $62 \%$ of them strongly agree that Strategic FM helps to prioritise FM needs. In spite of this, majority of them (62\%) stated that their companies do not adhere to Strategic FM standards or guidelines when practicing FM as shown in Table 1.0.

Table 1: Results on variables tested for Strategic FM

\begin{tabular}{lrc}
\hline Variables & \multicolumn{2}{c}{ Highest Selection (\%) } \\
\hline Strategic FM improves our service quality & Agree & $69 \%$ \\
Strategic FM helps to prioritise FM needs & Strongly Agree & $62 \%$ \\
My company adheres to Strategic FM standards/guidelines when & Disagree & $62 \%$ \\
practising FM & \\
\hline
\end{tabular}

Pearson Chi-square test was used to analyse the differences within the organisation types on the implementation of FM Strategies ( $\mathrm{p}>0.005)$. In summary, more than half of the respondents from the majority of large companies do not have FM strategies (Refer Table 2). For those that do have FM strategies, they are mainly from medium and large size companies and mostly have been using them for more than 5 years. All FM practitioners from this group also agree that FM strategies help to enhance their service delivery and $56 \%$ of them believe that FM strategies contribute to the success of their clients' corporate missions. On the other hand, a small fraction (13\%) of them signifies that the FM strategies practised do not integrate with their clients' corporate objectives/ strategies. This accords closely with Barret [29], where he stated that there is a gap that fails to link FM strategies with client's strategies and FM companies have to rectify the situation in order to deliver a service that meets the clients' objectives.

Table 2: Results on the implementation of FM strategies

\begin{tabular}{cccccc}
\hline Does your company have any FM Strategies? $(n=35)$ & & Yes & & No & Not Sure \\
\hline Organisation Type & Large & 7 & 9 & 1 \\
& Medium & 8 & 5 & 1 \\
& Small & 1 & 3 & 0 \\
& Total & 16 & 17 & 2 \\
\hline
\end{tabular}

\subsection{Performance Measurement System (PMS) in FM}

It was found that a large amount of respondents $(60 \%)$ have no implementation of Performance Measurement System (PMS) for their FM service delivery as indicated in Table 3. One survey participant responded that the reason why PMS is not implemented is to avoid a high key performance indicator (KPI) in the service performance.

Table 3: Results on the implementation of PMS for FM

\begin{tabular}{cc}
\hline Does your company implement any PMS for FM? & Percentage (n=35) \\
\hline Yes & $40 \%$ \\
No & $60 \%$ \\
\hline
\end{tabular}

Further analysis on the respondents that implement PMS shows that half of the respondents $(50 \%)$ agree that their PMS is in line with the client's corporate objectives (Refer Table 4). Similarly, 
$43 \%$ of them agree that their performance measures are designed based on the FM strategies and that the FM performance has improved since the implementation of the PMS.

Table 4: Results on respondents' level of agreements on the PMS implementation and improvement

\begin{tabular}{llrc}
\hline Measurement & Variables & Highest Selection (\%) \\
& & & \\
\hline Implementation & Our PMS is designed based on the FM strategies & Agree & $43 \%$ \\
& Our PMS is in line with the client's corporate objectives & Neutral & $50 \%$ \\
Improvement & Our FM performance has improved since the implementation of & Agree & $43 \%$ \\
& the PMS & & \\
\hline
\end{tabular}

\subsection{Barriers/Problems in Implementing PMS}

Respondents were also asked to select the problems that they face in implementing PMS or for those that do not implement PMS, the barriers that they perceive are the potentially hindering the implementation of PMS in the FM service. Table 5 shows the list of barriers/ problems in implementing PMS based on the ranks voted by the respondents. The proposed variables are derived from literature review and categorised into 4 elements namely Management, Employee, Formulation of Measures and also the Service Direction.

Table 5: Problems or barriers in implementing PMS

\begin{tabular}{cll}
\hline Rank & Variables & Problems/ Barriers in Implementing PMS \\
\hline 1 & Service Direction & - More focused on short-term decision making \\
2 & Service Direction & - Rely on software as solution \\
3 & Service Direction & - Lack of strategic planning skill \\
4 & Formulation & - No proper sample or guidance \\
5 & Formulation & - Problems identifying suitable measures \\
6 & Formulation & - Difficulties in evaluating the relative importance of measures \\
7 & Service Direction & - Lack of awareness and understanding on the importance \\
8 & Management & - Unclear of objectives and benefits \\
9 & Management & - Inadequate training and support \\
10 & Management & - Manager's resistance \\
11 & Management & - Lack of senior management commitment \\
12 & Employee & - Employee resistance to change \\
13 & Employee & - Staff turnover and problems in reallocating new roles \\
14 & Employee & - Reluctant to invest time and energy \\
15 & Employee & - Lack of clarity/rationale \\
16 & Formulation & - Striving for perfection \\
17 & Formulation & - Unclear of the process and procedure involved \\
18 & Employee & - Employee fear of status affected or stressful work conditions \\
19 & Management & - Organizational fears \\
20 & Service Direction & - Priorities in other management aspect \\
21 & Others & - Time and energy consuming for managers to focus on \\
& [Management] & - Burdened with current workload \\
& & - Need support from top management \\
& [Employee] & - Time consuming to train employees \\
& [Formulation] & - Do not know where to start/ starting point \\
& [Service Direction] & - Lack of resources \\
& & - Not clear of the long-term goal \\
\hline
\end{tabular}

Results from Table 5 signify that the top three problems identified in the implementation of PMS are rooted in Service Direction where most of the respondents are more focused on the short- 
term decision-making or goal only rather than considering the long-term goal. They were also more reliant on the software tool as the problem solution and most importantly they believed that lack of strategic planning skill in planning for the performance monitoring and evaluation is also the problem and barrier in implementing PMS. The respondents also provided some problems that are not listed but which they believed are among the contributing factors, for instance they believed that it is both time- and energy-consuming for managers to focus on the implementation of PMS and also to train employees. They also believed that they were much burdened with current workload and PMS is not something that they would want to look into. They further believed that the top management needs to give full support for the PMS implementation to ensure its effectiveness.

As for the formulation of measures, they had no idea of where to start and how to work on designing a PMS considering the lack of resources that they have. One of their responses was also that they were not clear regarding the long-term goal of PMS and the long-term benefits behind its implementation. In general, it can be seen that the lack of strategic planning, service direction and management skills and goals are the factors that hamper the implementation of PMS for FM in Malaysia. The scenario fits with the slow progress of FM development in Malaysia, where there is a lack of guidelines and procedures towards enhancing standard service delivery. The implementation of PMS as a strategic step forward is considered new and alien to the FM service sectors there, as the industry is still immature.

\section{Conclusions}

The overall survey findings show that FM industry in Malaysia is still not fully developed and yet to be up to par with FM industries in other countries. With majority of the FM practitioners are still implementing operational FM and no performance measurement system in their practice, the future of this industry in Malaysia is hard to be predicted. The industry appears to be passive as there is little development in FM industry since the first statement in 2001 by the then Deputy Prime Minister of Malaysia Datuk Seri Abdullah Badawi on the need of change in the providing good services and improve the upkeep of buildings. Similarly, the implementation of PMS is relatively low among the FM practitioners. In spite of that, the practitioners that implement PMS understood the importance of having the PMS that is line with the FM strategies and corporate objectives. They also show positive feedback in seeing the improvement and benefits with the practice of PMS in their daily service delivery. Based on the results derived from the pilot study, this research will take a step forward in exploring the appropriate performance measures for FM targeting for each FM service aspect. This study will also look at the possibility in designing and developing a performance measures framework based on the literature review and data collected from FM service companies in UK where the industry is progressive. The PMS Framework in FM that will be developed at the end of this research is anticipated to improve the current development of FM in Malaysia particularly in the context of PM implementation and FM profession and service delivery.

\section{Acknowledgement}

Special thanks to Prof Michael Pitt from University College London; Bartlett School of Graduate Studies, University College London; University of Malaya; and Ministry of Higher Education for the cooperation and arrangements made in conjunction with the conduct of this research. The cooperation given by all the FM practitioners participating in this research is highly appreciated. 


\section{References}

1. Neely, A., Gregory,M. and Platts,K. (1995). Performance measurement system design: a literature review and research agenda. International Journal of Operations \& Production Management, Vol. 15 No.4, pp.80-116.

2. Parida, A. and Kumar,U. (2006). Maintenance Performance Measurement (MPM): Issues and Challenges. Journal of Quality in Maintenance, Vol. 12 No.3 pp.239-251.

3. Bititci, U., Carrie, A. and McDevitt, L. (1997). Integrated performance measurement systems: a development guide. International Journal of Operations \& Management, Vol. 17 No.5, pp. 522534.

4. Kagioglou, M., Cooper, R. and Aouad, G. (2001). Performance management in construction: a conceptual framework. Construction Management \& Economics, 19, 85- 95.

5. Parker, C. (2000). Performance Measurement. Work study, 49 (2), 63-66.

6. Martinez, V. (2005). Performance measurement systems: mix effects. http://euram2005.wi.tum.de [Accessed on: 18 May 2011.

7. Baldwin, A., McCaffer, R. and Osman, I. I. (2001). Project performance in a contracting organisation: Analysis, evaluation and development. Proceedings of CIB World Congress, Willington, New Zealand.

8. Tangen, S. (2003). An Overview of Frequently used Performance Measures. Work Study, Vol. 52 No.7, pp. 347-354.

9. Amaratunga, D. (2000). Assessment of Facilities Management Performance. Property Management, Vol. 18 No. 4, pp. 258-266.

10. Meekings, A. (1995). Unlocking the potential of performance measurement: a practical implementation guide. Public Money and Management, Vol. 15 No. 4, p5-12

11. Cain, C. T. (2004). Performance Measurement for Construction Profitability. Blackwell Publishing Ltd., Oxford

12. Nanni, A.J., Dixon, J.R. and Vollmann, T.E. (1992). Integrated performance measurement: management accounting to support the new manufacturing realities. Journal of Management Accounting Research, Vol. 4, pp. 1-19.

13. Rouse, P. and Putterill, M., (2003). An integral framework for performance measurement. Management Decision Vol. 41 No. 8, pp. 791-805.

14. Tangen, S. (2002). A theoretical foundation for productivity measurement and improvement of automatic assembly systems. Licentiate thesis, The Royal Institue of Technology, Stockholm

15. De Toni, A.F., Fornasier, A. Montagner, M. and Nonino, F. (2007). A performance measurement system for facility management: the case study of a medical service authority. International Journal of Productivity and Performance Management, Vol. 56, Nos. 5/6, pp. 417-435.

16. Rose, K.H. (1995). A performance measurement model. Quality Progress, February, pp. 63-66.

17. Williams, B. (1993). What a performance!. Property Management, Vol. 11, No. 3, pp. 190-199.

18. Rummler, G.A. and Brache, A.P. (1995). Improving Performance: How to Manage the White Space on the Organisation Chart. San Francisco: Jossey-Bass Publishers.

19. Pintelon, L. and Van Puyvelde, F. (1997). Maintenance performance reporting systems: some experiences. Journal of Quality in Maintenance Engineering, Vol. 3 No. 1, pp.4 - 15

20. Kennerly, M. and Neely, A. (2003). Measuring Performance in A Changing Business Environment. International Journal of Operation \& Production Management, Vol. 23 No. 2, pp. 213-29

21. Lingle, J. H. and Schiemann, W. A. (1996). From balanced scorecard to strategy gauge. Is measurement worth it?. Management Review, March, pp. 56-62.

22. Baharum, M.R. and Pitt, M. (2009). Determining a conceptual framework for green FM intellectual capital. Journal of Facilities Management, Vol. 7 No. 4, pp. 267-282.

23. Becker, F. (1990). The total workplace. Van Nostrand Reinhold, New York, NY

24. Amaratunga, D. and Baldry, D. (2000). Assessment of facilities management performance in higher education properties. Facilities, No. 7/8, pp. 293-301. 
25. Hudson, M., Smart, A. and Bourne, M. (2001). Theory and practice in SME performance measurement systems. International Journal of Operations \& Production Management, Vol. 21 No.8, pp. 1096-1115.

26. Kadefors, A. (2008). Contracting in FM: collaboration, coordination and control. Journal of Facilities Management, Vol. 6 No. 3, pp. 178-188.

27. Enoma, A. and Allen, S. (2007). Developing key performance indicators for airport safety and security. Facilities, Vol. 25 No. 7, pp. 296-315.

28. McDougall, G. and Hinks, J. (2000). Identifying priority issues in facilities management benchmarking. Facilities, Vol. 18 No. 10/11/12, pp. 427-434

29. Becker, F. (1990). The total workplace. Van Nostrand Reinhold, New York, NY 